\title{
Cumulative Distribution Functions of Sildenafil Citrate on Exercise Capacity and Hemodynamic Function in Children with Pulmonary Arterial Hypertension
}

Joseph C Cappelleri' ${ }^{*}$, Lie-Ju Hwang ${ }^{2}$, Jack Mardekian ${ }^{2}$, Simon A Teal $^{3}$, and Marko A Mychaskiw ${ }^{4}$

${ }^{1}$ Pfizer Inc, Groton, CT, USA

${ }^{2}$ Pfizer Inc, New York, NY, USA

${ }^{3}$ Pfizer Ltd, Tadworth, UK

${ }^{4}$ Pfizer Inc, Collegeville, PA, USA

\section{Abstract}

Background: Pulmonary Arterial Hypertension (PAH) is an ultimately fatal condition characterized by reduced exercise capacity and impaired hemodynamic parameters. Although these relationships are well described in adults with $\mathrm{PAH}$, they are not characterized in pediatric populations.

Methods: Children (aged 1-17 years) with PAH received 16 weeks of randomized, double-blind treatment with sildenafil or placebo. The primary outcome measure, peak $\mathrm{VO}_{2}\left(\mathrm{PVO}_{2}\right)$, was assessed using cardiopulmonary exercise testing in children developmentally capable of exercise. Secondary measures (for all patients) included Mean Pulmonary Arterial Pressure (mPAP), Pulmonary Vascular Resistance Index (PVRI), and cardiac index. Cumulative Distribution Functions (CDFs) plotted the cumulative percentage of patients against percentage changes (and also numeric changes) from baseline to week 16 in outcome measures. The Kolmogorov-Smirnov test assessed the difference in separation between sildenafil CDF and placebo CDF.

Results: Of 234 randomized and treated children, 106 were developmentally able to exercise and 148 to 221 provided non-exercise endpoint data. Significant differences between the CDFs were observed for sildenafil (all doses combined) vs. placebo for percentage changes in $\mathrm{PVO}{ }_{2}$, mPAP, and PVRI $(P=0.02,0.01$, and 0.001 , respectively) but not for cardiac index $(P=0.14)$. Fifty-eight of $77(75 \%)$ sildenafil-treated children had increases in the percentage change in $\mathrm{PVO}_{2}$ vs. 13 of 29 (45\%) placebo-treated children. Across all measures, a similar pattern of results was obtained when numeric (rather than percentage) changes were assessed.

Conclusions: Cumulative distribution functions, which incorporate the entire distribution of responses, can enhance clinical interpretation of outcome measures. By CDF analysis, an enhanced profile of sildenafil (vs. placebo) was observed, indicating improvement in $\mathrm{PVO}_{2}, \mathrm{mPAP}$ and PVRI, but not cardiac index, in children with $\mathrm{PAH}$.

Keywords: Pulmonary arterial hypertension; Pediatric; Exercise capacity; Hemodynamics; Cumulative distribution function; Outcome

Abbreviations: 6MWD: 6-Minute Walk Distance; 6MWT: 6-Minute Walk Test; CPET: Cardiopulmonary Exercise Testing; CHD: Congenital Heart Disease; CDF: Cumulative Distribution Function; cGMP: Cyclic Guanosine Monophosphate; EMA: European Medicines Agency; FDA: US Food and Drug Administration; HRQoL: Health-Related Quality of Life; mPAP: Mean Pulmonary Arterial Pressure; $\mathrm{PVO}_{z}:$ Peak $\mathrm{VO}_{2}$; PPHN: Persistent Pulmonary Hypertension of the Newborn; PDE-5: Phosphodiesterase-type 5; PAH: Pulmonary Arterial Hypertension; PVR: Pulmonary Vascular Resistance; PVRI: Pulmonary Vascular Resistance Index; WHO: World Health Organization

\section{Introduction}

Pulmonary Arterial Hypertension (PAH) is a rare and devastating condition characterized by a sustained increase in Pulmonary Vascular Resistance (PVR) leading to right ventricular failure and premature death [1]. Patients with PAH have decreased physical functioning [2] and decreased Health-Related Quality Of Life (HRQoL) [3,4], as well as reduced survival [5].

As a rare disease, PAH has an estimated prevalence of 15 to 26 cases per million in adults [6,7]. In children, PAH may be even less common, with an estimated prevalence of PAH (excluding Persistent Pulmonary Hypertension of the Newborn [PPHN] and PAH caused by congenital heart disease [PAH-CHD]) of 3.7 per million children based on a registry in France [8]. However, because PAH-CHD represents a large proportion of cases (up to 40\%) [9], this figure is likely to be underestimated.
While there are differences in the characteristics of PAH in children and adults, most notably disease etiology, presenting symptoms and hemodynamic profile [10], the pathophysiological mechanisms and clinical presentation are similar. Consequently, expert opinion states that the treatment guidelines outlining diagnostic work-up and therapeutic algorithms for adults may also be applied, with caution, to children with PAH $[11,12]$.

A number of therapies are approved and available for the treatment of adult $\mathrm{PAH}$, including prostacyclin analogues (epoprostenol, treprostinil, iloprost), endothelin receptor antagonists (bosentan, ambrisentan), and phosphodiesterase-type 5 (PDE-5) inhibitors (sildenafil, tadalafil). There are, however, limited data on the use of these medications in children.

The PDE-5 class is the predominant PDE isoform in the lung that metabolizes cyclic guanosine monophosphate (cGMP), the second messenger of the potent endogenous vasodilator nitric

*Corresponding author: Joseph C Cappelleri, Pfizer Inc, 445 Eastern Point Road MS 8260-2502, Groton, CT, USA 06340, Tel: +1 860441 8033; Fax: +1 860686 5139; E-mail: joseph.c.cappelleri@pfizer.com

Received March 26, 2013; Accepted April 20, 2013; Published April 22, 2013

Citation: Cappelleri JC, Hwang LJ, Mardekian J, Teal SA, Mychaskiw MA (2013) Cumulative Distribution Functions of Sildenafil Citrate on Exercise Capacity and Hemodynamic Function in Children with Pulmonary Arterial Hypertension. J Pulmon Resp Med S4: 002. doi:10.4172/2161-105X.S4-002

Copyright: (C) 2013 Cappelleri JC, et al. This is an open-access article distributed under the terms of the Creative Commons Attribution License, which permits unrestricted use, distribution, and reproduction in any medium, provided the original author and source are credited. 
oxide. By selectively inhibiting PDE-5, sildenafil citrate promotes the accumulation of intracellular cGMP and thereby enhances nitric oxide-mediated vasodilatation [13].

Sildenafil has been studied in two randomized, double-blind, placebo-controlled studies in adults with PAH who were treatmentnaïve (SUPER-1) [13] and those who were already being treated with intravenous epoprostenol therapy (PACES-1) [14]. Greater improvements in exercise capacity, hemodynamic parameters, and HRQoL (assessed using the Short Form [SF]-36) were observed in patients treated with sildenafil when compared with placebo after 12 (SUPER-1) or 16 (PACES-1) weeks. Sildenafil (Revatio ${ }^{\circledR}$ ) was approved for the treatment of adults with PAH (for the indication to improve exercise capacity) in 2005 in the US and Europe; in 2009, the indication to delay clinical worsening was approved (US only) $[15,16]$.

The effect of sildenafil on children in PAH was evaluated in a 16-week, placebo-controlled, dose-ranging study (STARTS-1) [17]. Sildenafil monotherapy was well tolerated over 16 weeks, and improvements were demonstrated in exercise capacity, functional class and hemodynamics. Based on the results of that study, the European Medicines Agency (EMA) approved sildenafil in 2010 for the treatment of children (aged 1-17 years) with PAH [15]. In 2012 the regulatory filing was rejected by the US Food and Drug Administration (FDA).

Often, because of lack of sufficient history or knowledge with the measure, it can be difficult to interpret the clinical meaning or meaningful import of statistically significant changes in outcome scores. As one way to address the issue, responder analyses have been proposed by having a threshold or cutoff score to indicate whether a change in a patient score has clinical meaning or, at least, a meaningful interpretation $[18,19]$. A responder analysis is based on a binary outcome (yes or no) of whether a patient achieved a particular efficacy threshold (e.g., at least a $10 \%$ improvement from baseline to followup) and consequently qualified as a responder. However, a major consideration with responder analysis is often the arbitrary nature of defining the threshold for a response $[20,21]$.

Typically, treatment effects in randomized controlled trials in patients with $\mathrm{PAH}$ have been reported using summary or grouplevel estimates to indicate improvements from baseline achieved with active treatment group compared with that achieved with placebo $[13,14]$. As a supplement to mainstream group-level statistics and, if conducted, responder analyses, Cumulative Distribution Functions (CDFs) can be used to augment such summary-level results (and responder analyses, if performed) for quantitative outcomes, be they objectively or subjectively assessed $[18,19,22,23]$. Cumulative distribution functions display patient response rates over a continuum of possible thresholds, thus eliminating any arbitrariness associated with a particular threshold definition for a responder. Such a CDF, one for each treatment group, would allow for a variety of response thresholds to be examined simultaneously and would encompass all data. While their descriptive richness is undeniable, and despite their not infrequent use in pulmonary and respiratory areas [24,25], their underuse is also undeniable.

Cumulative distribution functions can lend clinical relevance and enhance interpretation of treatment differences on outcomes for regulatory and scientific purposes. In this article we report and describe CDFs on exercise capacity and hemodynamic parameters in children with PAH treated with sildenafil in the STARTS-1 study [17].

\section{Materials and Methods}

Response profiles on CDFs for exercise capacity and hemodynamic function were generated using data from the STARTS- 1 clinical trial in which children aged 1-17 years with PAH received 16 weeks of randomized, double-blind treatment with sildenafil or placebo. A full description of the STARTS-1 clinical trial can be found elsewhere [17]

Randomization in this clinical trial was stratified by weight and developmental ability to perform cardiopulmonary exercise testing (CPET; assessed using bicycle ergometry). Patients $>20 \mathrm{~kg}$ were randomized 1:1:1:1 to placebo and sildenafil low-, medium-, and highdose groups, respectively; patients $8-20 \mathrm{~kg}$ were randomized to 1:2:1 to placebo and sildenafil medium- and high-dose groups.

The primary outcome measure of peak $\mathrm{VO}_{2}\left(\mathrm{PVO}_{2}\right)$ was assessed by CPET in children who were developmentally capable of exercise at baseline and at week 16. Secondary outcome measures included mean Pulmonary Arterial Pressure (mPAP), Pulmonary Vascular Resistance Index (PVRI) and cardiac index, all of which were assessed at baseline and at week 16 in all patients. Last-observation-carried-forward values were used in all presentations and analyses of numeric change from baseline (i.e., week 16 score minus baseline score) and percentage change from baseline (i.e., [week 16 score minus baseline score] divided by baseline score).

Baseline characteristics were summarized for all patients. Baseline characteristics of categorical variables were summarized by placebo and sildenafil citrate dose using frequencies and percentage in each category. Means and standard deviations were reported for continuous baseline characteristics.

Cumulative distribution functions depict the probability of a variable having values less than or equal to a particular value and do so across each particular value of the variable; alternatively, 1 minus that probability is the probability of a variable having values greater than a particular value. Cumulative distribution functions were generated using data from all children who were developmentally capable. A continuous plot of the percentage (and, separately, also numeric) change from baseline was presented on the horizontal axis and the cumulative percentage of patients experiencing that change or less presented on the vertical axis. These plots depicted the cumulative percentage of patients on the vertical axis against percentage changes (and also numeric changes) from baseline to week 16 in outcome measures on the horizontal axis.

Regarding the outcome measures, increases in $\mathrm{PVO}_{2}$ and cardiac index are beneficial, while decreases in MPAP and PVRI are beneficial Cumulative distribution functions of sildenafil dose (low, medium, high) groups were compared over all dose groups and also by each dose group relative to placebo using the Kolmogorov-Smirnov nonparametric test of equality between two distributions [22]. A two-sided $\boldsymbol{p}$-value $\leq 0.05$ was considered statistically significant for descriptive purposes in all analyses. No adjustments were made for multiple pairwise comparisons.

All CDFs were generated and all analyses were conducted using SAS 9.2 (Statistical Analysis System, Cary, North Carolina).

\section{Results}

\section{Patient population}

A detailed description of the patient population appears in the main clinical publication [17]. Here we highlight several baseline patient characteristics (Table 1). Of 234 patients randomized and treated, $33 \%$ had idiopathic or heritable $\mathrm{PAH}$; the remaining $67 \%$ had PAH-CHD. Across the three sildenafil dose groups, comparability was found with respect to etiology, baseline World Health Organization 
Citation: Cappelleri JC, Hwang LJ, Mardekian J, Teal SA, Mychaskiw MA (2013) Cumulative Distribution Functions of Sildenafil Citrate on Exercise Capacity and Hemodynamic Function in Children with Pulmonary Arterial Hypertension. J Pulmon Resp Med S4: 002. doi:10.4172/2161105X.S4-002

Page 3 of 8

(WHO) functional class, peak oxygen consumption, mean pulmonary artery pressure, and pulmonary vascular resistance index. The placebo group appeared numerically to have less severe disease (assessed by pulmonary oxygen consumption, hemodynamics, and WHO functional class). Cardiopulmonary exercise testing was performed in 115 developmentally able patients; of them, 106 were evaluable for analysis on peak oxygen consumption (Table 1).

\section{Outcomes}

As reported and detailed previously [17], the estimated mean

\begin{tabular}{|c|c|c|c|c|c|}
\hline & & \multicolumn{4}{|c|}{ Sildenafil Dose } \\
\hline & $\begin{array}{c}\text { Placebo } \\
(n=60)\end{array}$ & $\begin{array}{l}\text { Low } \\
(n=42)\end{array}$ & $\begin{array}{c}\text { Medium } \\
(n=55)\end{array}$ & $\begin{array}{l}\text { High } \\
(n=77)\end{array}$ & $\begin{array}{c}\text { Combined } \\
(n=174)\end{array}$ \\
\hline Female sex, $\mathrm{n}(\%)$ & $38(63)$ & $25(60)$ & $31(56)$ & $51(66)$ & $107(62)$ \\
\hline \multicolumn{6}{|l|}{ Age in years, $\mathrm{n}(\%)$} \\
\hline $1-4$ & $7(11)$ & $0(0)$ & $9(16)$ & $19(25)$ & $28(16)$ \\
\hline $5-12$ & $37(62)$ & $25(60)$ & $28(51)$ & $36(47)$ & $89(51)$ \\
\hline $13-17$ & $16(27)$ & $17(40)$ & $18(33)$ & $22(29)$ & $57(33)$ \\
\hline \multicolumn{6}{|l|}{ WHO functional class, n (\%) } \\
\hline I & $25(42)$ & $9(21)$ & $20(36)$ & $21(27)$ & $50(29)$ \\
\hline II & $29(48)$ & $23(55)$ & $25(45)$ & $43(56)$ & $91(52)$ \\
\hline III & $6(10)$ & $9(21)$ & $8(15)$ & $12(16)$ & $29(17)$ \\
\hline IV & 0 & 0 & $1(2)$ & 0 & $1(1)$ \\
\hline Missing & 0 & $1(2)$ & $1(2)$ & $1(1)$ & $3(2)$ \\
\hline \multicolumn{6}{|l|}{ Etiology, n (\%) } \\
\hline IPAH/FPAH & $21(35)$ & $12(29)$ & $19(35)$ & $26(34)$ & $57(33)$ \\
\hline PAH-CHD & $39(65)$ & $30(71)$ & $36(65)$ & $51(66)$ & $117(67)$ \\
\hline \multicolumn{6}{|l|}{ PAH-related variables, mean (SD) } \\
\hline Peak VO${ }_{2}, \mathrm{~mL} / \mathrm{kg} / \mathrm{min}^{\dagger}$ & $20(4)$ & $18(4)$ & $18(5)$ & $17(4)$ & $18(4)$ \\
\hline Mean pulmonary artery pressure, $\mathrm{mmHg}^{\ddagger}$ & $59(22)$ & $66(23)$ & $62(18)$ & $62(24)$ & $63(22)$ \\
\hline Cardiac index, $\mathrm{L} / \mathrm{min} / \mathrm{m}^{2 \S}$ & $3.9(2.1)$ & $3.1(1.1)$ & $3.3(1.5)$ & $3.4(1.6)$ & $3.3(1.5)$ \\
\hline Pulmonary vascular resistance index, Wood units $\bullet \mathrm{m}^{2 \|}$ & $15(10)$ & $22(13)$ & $19(14)$ & $20(16)$ & $20(15)$ \\
\hline
\end{tabular}

Note: $\mathrm{CHD}=$ congenital heart disease; $\mathrm{FPAH}=$ familial $\mathrm{PAH}$; IPAH=idiopathic $\mathrm{PAH}$; $\mathrm{PAH}=$ pulmonary arterial hypertension; $\mathrm{VO}_{2}=\mathrm{oxygen}$ consumption; $\mathrm{WHO}_{\mathrm{H}}=\mathrm{World}_{\mathrm{H}} \mathrm{Health}$ Organization.

*The groups shown represent all treated patients.

tSubset of patients developmentally able to perform exercise testing ( $n=30,28,28,29$, and 85 for placebo, sildenafil low-, medium-, and high-dose groups, and sildenafil combined dose group, respectively).

$\ddagger_{n}=59,42,55,75$, and 172 for placebo, sildenafil low-, medium-, and high-dose groups, and sildenafil combined dose group, respectively. $\S_{n}=59,41,52,74$, and 167 for placebo, sildenafil low-, medium-, and high-dose groups, and sildenafil combined dose group, respectively. $\|_{n}=57,40,52,73$, and 165 for placebo, sildenafil low-, medium-, and high-dose groups, and sildenafil combined dose group, respectively. Table 1: Baseline Patient Characteristics from STARTS-1*

\begin{tabular}{|c|c|c|c|c|}
\hline \multirow[b]{2}{*}{ Statistic } & \multicolumn{4}{|c|}{ Treatment Difference (Sildenafil - Placebo) } \\
\hline & Sildenafil Low Dose & Sildenafil Medium Dose & Sildenafil High Dose & Sildenafil Combined Dose \\
\hline Mean PAP (mmHg) & $\mathrm{n}=39$ & $\mathrm{n}=55$ & $\mathrm{n}=71$ & $n=165$ \\
\hline Mean difference \pm SE & $1.6 \pm 3.1$ & $-3.5 \pm 2.7$ & $-7.3 \pm 2.6$ & $-3.1 \pm 2.2$ \\
\hline $95 \% \mathrm{Cl}$ & $-4.5,7.6$ & $-8.9,1.9$ & $-12.4,-2.1$ & $-7.5,1.3$ \\
\hline$P$ value* & 0.610 & 0.199 & 0.006 & 0.172 \\
\hline PVRI (Wood units•m²) & $\mathrm{n}=37$ & $\mathrm{n}=51$ & $\mathrm{n}=68$ & $n=156$ \\
\hline Ratio $^{+}$ & 0.982 & 0.819 & 0.727 & 0.836 \\
\hline $95 \% \mathrm{Cl}$ & $0.802,1.203$ & $0.684,0.981$ & $0.612,0.863$ & $0.720,0.971$ \\
\hline$P$ value* & 0.859 & 0.031 & $<0.001$ & 0.019 \\
\hline Cardiac index $\left(\mathrm{L} / \mathrm{min} / \mathrm{m}^{2}\right)$ & $\mathrm{n}=37$ & $\mathrm{n}=51$ & $\mathrm{n}=69$ & $n=157$ \\
\hline Ratio $^{\dagger}$ & 1.100 & 1.043 & 1.148 & 1.096 \\
\hline $95 \% \mathrm{Cl}$ & $0.963,1.258$ & $0.925,1.176$ & $1.026,1.286$ & $0.994,1.210$ \\
\hline$P$ value* & 0.161 & 0.486 & 0.017 & 0.066 \\
\hline
\end{tabular}

$\mathrm{Cl}=$ confidence interval; PVRI=pulmonary vascular resistance index; PAP=pulmonary artery pressure; STARTS-1=Sildenafil in Treatment-Naïve Children, Aged 1 to 17 Years, With Pulmonary Arterial Hypertension.

*With the exception of the primary comparison, $P$ values should be interpreted descriptively because no adjustments were made for multiple comparisons. †Because PVRI and cardiac index data were log-transformed before analysis, comparisons are presented as ratios (active/placebo) when back-transformed. $\mathrm{N}=56,52$ and 55 for the placebo group for mean PAP, PVRI, and cardiac index, respectively.

Table 2: Placebo-Corrected Change in Hemodynamic Outcomes Between Baseline and End of Double-Blind Treatment in STARTS-1. 
Citation: Cappelleri JC, Hwang LJ, Mardekian J, Teal SA, Mychaskiw MA (2013) Cumulative Distribution Functions of Sildenafil Citrate on Exercise Capacity and Hemodynamic Function in Children with Pulmonary Arterial Hypertension. J Pulmon Resp Med S4: 002. doi:10.4172/2161105X.S4-002

percentage change \pm standard error $\mathrm{PVO}_{2}$ for the three doses combined versus placebo was $7.7 \% \pm 4.0 \%$ [95\% Confidence Interval $(\mathrm{CI}),-0.2 \%$ to $15.6 \% ; P=0.056]$. Placebo-corrected estimates were made for the lowdose $(3.8 \% \pm 5.0 \%$ [95\% CI, $-6.1 \%$ to $13.7 \%])$, medium-dose $(11.3 \%$ $\pm 4.8 \%$ [95\% CI, $1.7 \%$ to $20.9 \%])$, and high-dose $(8.0 \% \pm 4.9 \%[95 \%$ $\mathrm{CI},-1.6 \%$ to $17.6 \%]$ ) groups. Hemodynamic parameters improved with medium or high doses versus placebo; low-dose sildenafil was ineffective (Table 2).

No statistical or meaningful differences on the Child Health Questionnaire-Parent Form 28 were observed between the three sildenafil dose groups and placebo. A dose response was observed for functional class improvement. Compared with placebo, the odds ratios for functional class improvement were 0.6 (95\% CI, 0.2 to 2.0 ), 2.3 (95\% CI, 0.8 to 6.7), and 4.5 (95\% CI, 1.6 to 13.1) for sildenafil low-, medium-, and high-dose groups, respectively. Most adverse events were mild to moderate in severity.

\section{Cumulative distribution functions: Percentage change from baseline}

Figures 1-4 depict the percentage change from baseline in $\mathrm{PVO}_{2}$ and three hemodynamic parameters (mPAP, cardiac index) for placebo and sildenafil groups.

Descriptive separation between cumulative distribution functions was evident for sildenafil versus placebo for percentage changes in $\mathrm{PVO}_{2}$ (Figure 1, top panel). For example, 58 of 77 (75\%) sildenafiltreated children had increases in the percentage change (greater than no percentage change) in $\mathrm{PVO}_{2}$ versus 13 of 29 (45\%) placebo-treated children. Separation between sildenafil and placebo curves was evident for medium- and high-dose sildenafil groups (Figure 1, bottom panel; Table 3).
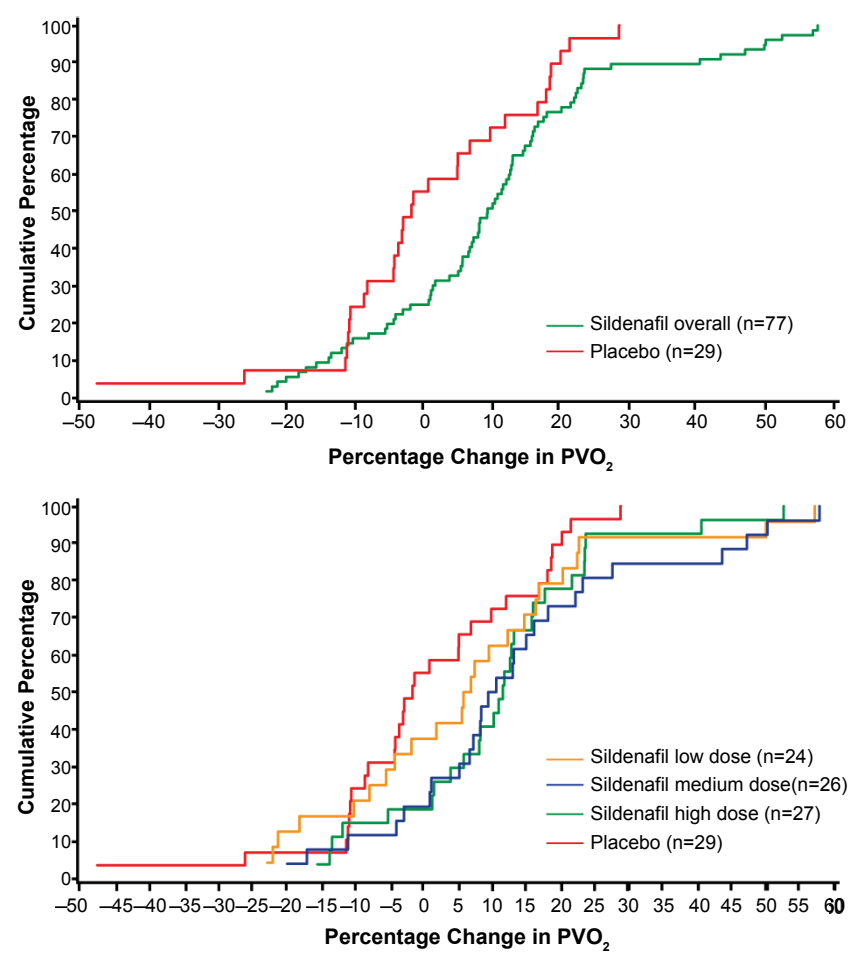

Figure 1: Cumulative Distribution Functions of Percentage Change from Baseline to Week 16 in Peak Volume $\mathrm{O}_{2}$ Consumption: Active Doses Combined vs. Placebo (Top) and Active Doses Separated vs. Placebo (Bottom).
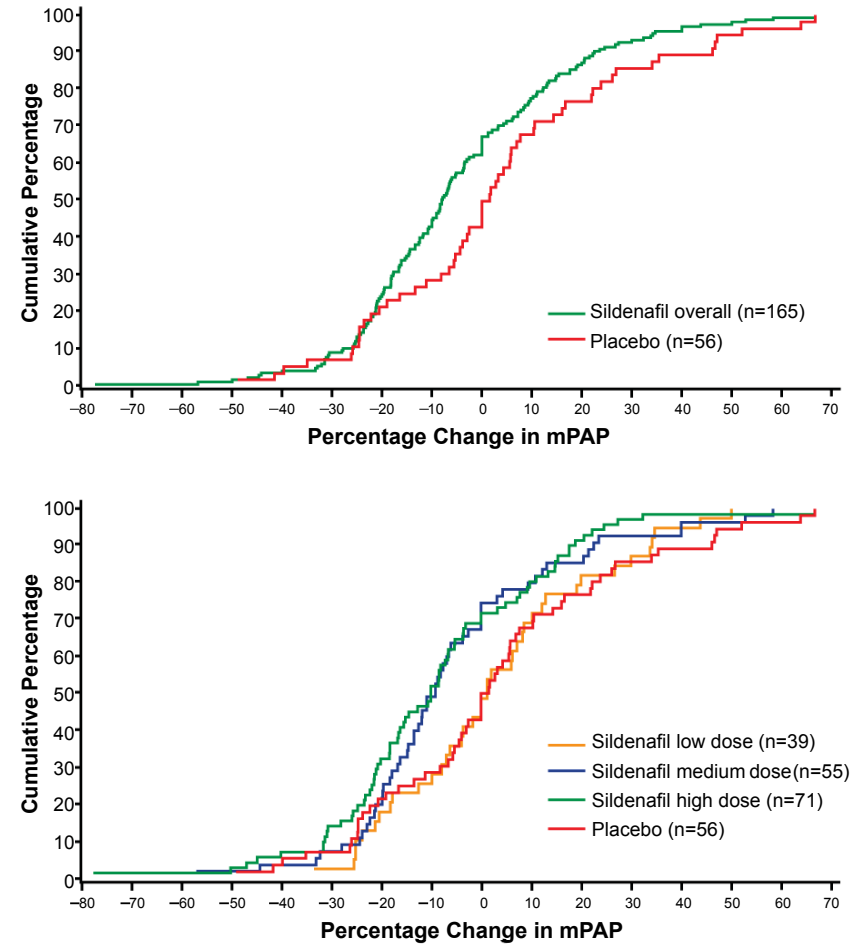

Figure 2: Cumulative Distribution Functions of Percentage Change from Baseline to Week 16 in Mean Pulmonary Arterial Pressure: Active Doses Combined vs. Placebo (Top) and Active Doses Separated vs. Placebo (Bottom).
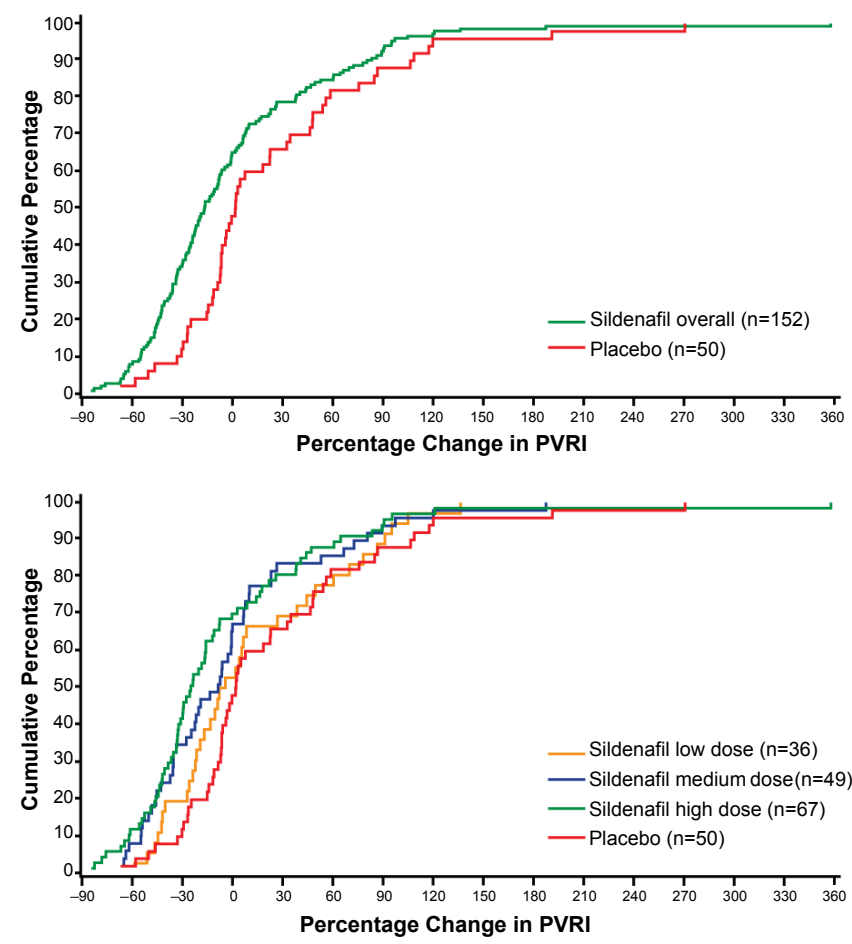

Figure 3: Cumulative Distribution Functions of Percentage Change from Baseline to Week 16 in Pulmonary Vascular Resistance Index: Active Doses Combined vs. Placebo (Top) and Active Doses Separated vs. Placebo (Bottom). 
Citation: Cappelleri JC, Hwang LJ, Mardekian J, Teal SA, Mychaskiw MA (2013) Cumulative Distribution Functions of Sildenafil Citrate on Exercise Capacity and Hemodynamic Function in Children with Pulmonary Arterial Hypertension. J Pulmon Resp Med S4: 002. doi:10.4172/2161105X.S4-002

Page 5 of 8

Descriptive separation between cumulative distribution curves was also evident for sildenafil versus placebo for percentage changes in the hemodynamic parameters of mPAP and pulmonary vascular resistance index, but not cardiac index (Figures 2-4). For mPAP, the CDFs for medium and high doses of sildenafil were manifest; for PVRI, only high-dose sildenafil distinguished itself from placebo (Table 3).
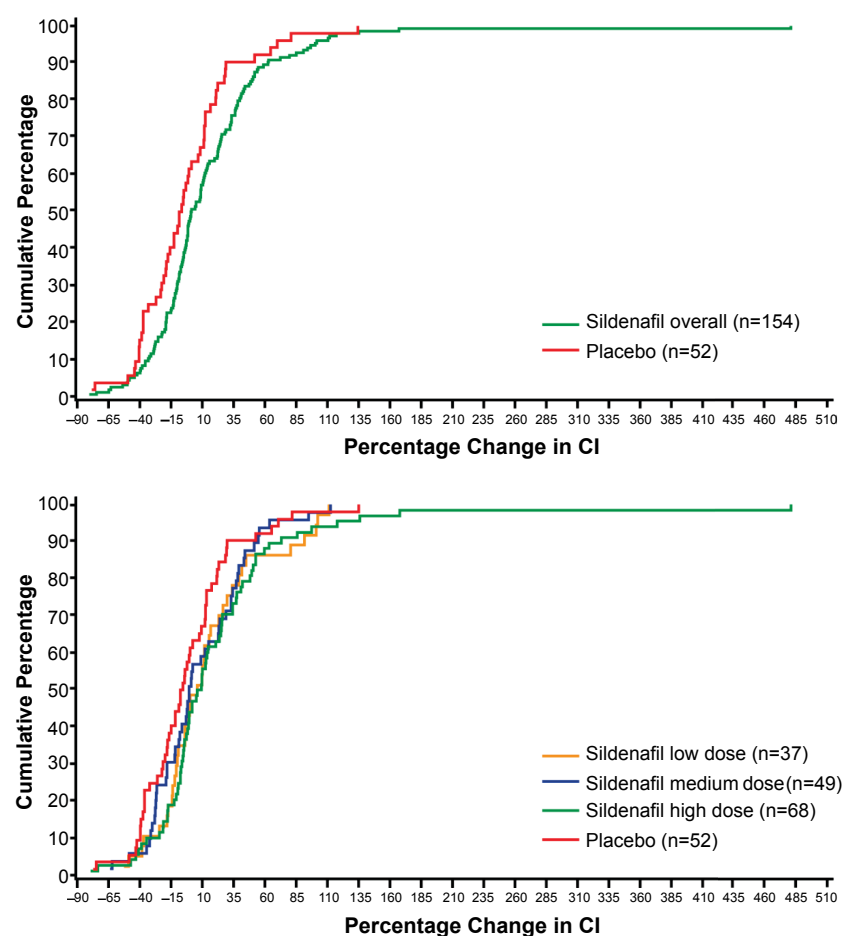

Figure 4: Cumulative Distribution Functions of Percentage Change from Baseline to Week 16 in Cardiac Index: Active Doses Combined vs. Placebo (Top) and Active Doses Separated vs. Placebo (Bottom).

\begin{tabular}{|l|c|c|c|c|}
\hline \multirow{2}{*}{ Outcome Measure } & \multicolumn{4}{|c|}{ Percentage Change: $\boldsymbol{P}$ Value* vs Placebo } \\
\cline { 2 - 5 } & Overall & Low & Medium & High \\
\hline Peak $\mathrm{VO}_{2}$ & $\mathbf{0 . 0 2}$ & 0.44 & $\mathbf{0 . 0 3}$ & $\mathbf{0 . 0 2}$ \\
\hline Mean PAP & $\mathbf{0 . 0 1}$ & 1.0 & $\mathbf{0 . 0 1}$ & $\mathbf{0 . 0 1}$ \\
\hline PVRI & $\mathbf{0 . 0 0 1}$ & 0.37 & 0.06 & $\mathbf{0 . 0 0 0 1}$ \\
\hline Cardiac index & 0.14 & 0.27 & 0.30 & 0.07 \\
\hline
\end{tabular}

$\mathrm{PAP}=$ pulmonary arterial pressure; $\mathrm{PVRI}=$ pulmonary vascular resistance index; $\mathrm{VO}_{2}=$ oxygen consumption.

* Values are rounded, with those $\leq 0.05$ in bold face.

Table 3: Descriptive $P$ Values (Active Treatment vs. Placebo) for Cumulative Distribution Functions: Percentage Change from Baseline.
Table 4, which is to be read in tandem with Figures 1-4, gives the percentage of patients in a given treatment group with percentage changes from baseline at or below a particular percentage change from baseline. Consider, for example, at most a $10 \%$ increase in $\mathrm{PVO}_{2}$ values from baseline to week 16 . Here $72.4 \%$ of patients met that criterion (the remaining $27.6 \%$ of patients had more than a $10 \%$ increase) in the placebo group, while $37.7 \%$ met that criterion (the remaining $62.3 \%$ of patients had more than a $10 \%$ increase) in the combined sildenafil groups. The percentages of patients in the combined sildenafil groups who had a zero or negative percentage change in $\mathrm{PVO}_{2}, \mathrm{mPAP}, \mathrm{PVRI}$ and cardiac index were $24.7 \%$ (vs. $55.2 \%$ placebo), $67.3 \%$ (vs. $50.0 \%$ placebo), $64.5 \%$ (vs. $48.0 \%$ placebo), and $47.4 \%$ (vs. $61.5 \%$ placebo), respectively.

\section{Cumulative distribution functions: Numeric change from baseline}

Across all four of these measures, a similar pattern of descriptive profiles to that of percentage changes emerged when numeric changes were assessed (Figures 5-8, Table 5).

Table 6, which is to be read in tandem with Figures 5-8, gives the percentage of patients in a given treatment group with numeric changes from baseline at or below a particular numeric change from baseline. Here 58.6\% of patients had at most a 1-point increase in $\mathrm{PVO}_{2}$ values (and the remaining $41.4 \%$ of patients had more than a 1-point increase) in the placebo group, while $37.7 \%$ met that criterion (and the remaining $62.3 \%$ of patients had more than a 1-point increase) in the combined sildenafil groups. The percentages of patients in the combined sildenafil groups who had a zero or negative numeric change in $\mathrm{PVO}_{2}$, mPAP, PVRI, and cardiac index were the same as the corresponding percentages with zero or negative percentage change.

\section{Discussion}

Pulmonary arterial hypertension is a rare, progressive and fatal disease. Effective therapies, first developed and approved in adults, are now being studied in children. Registration studies for $\mathrm{PAH}$ have traditionally used measures of exercise capacity as the primary endpoint, while hemodynamic measures have proved useful as secondary endpoints to assess impact on cardiopulmonary function. In adults, exercise capacity is assessed via the 6-minute walk test (6MWT). However, consistency, reproducibility and interpretability of the 6MWT are challenging in children [26,27]. In adults with PAH, 6-minute walk distance (6MWD) is known to correlate with $\mathrm{PVO}_{2}$ [28].

Therefore, in the STARTS-1 study the investigators selected to assess exercise capacity by measuring $\mathrm{PVO}_{2}$ during CPET [17]. In a post hoc analysis of data from the STARTS-1 study, in children who were developmentally and physically able to perform exercise testing, peak $\mathrm{VO}_{2}$ measurements exhibited good reliability, and improvements were associated with improvements in certain other relevant clinical

\begin{tabular}{|c|c|c|c|c|c|c|c|c|c|c|c|c|c|c|c|c|}
\hline \multirow[b]{2}{*}{ Percentage Change From Baseline } & \multicolumn{4}{|c|}{ Peak $\mathrm{VO}_{2}, \mathrm{~mL} / \mathrm{kg} / \mathrm{min}$} & \multicolumn{4}{|c|}{$\begin{array}{l}\text { Mean pulmonary arterial } \\
\text { pressure, } \mathrm{mmHg}\end{array}$} & \multicolumn{4}{|c|}{$\begin{array}{c}\text { Pulmonary vascular resistance } \\
\text { index, Wood units } \bullet m^{2}\end{array}$} & \multicolumn{4}{|c|}{ Cardiac index, $\mathrm{L} / \mathrm{min} / \mathrm{m}^{2}$} \\
\hline & $\mathbf{n}$ & $0 \%$ & $10 \%$ & $20 \%$ & $\mathbf{n}$ & $0 \%$ & $10 \%$ & $20 \%$ & $\mathbf{n}$ & $0 \%$ & $10 \%$ & $20 \%$ & $\mathbf{n}$ & $0 \%$ & $10 \%$ & $20 \%$ \\
\hline Placebo & 29 & 55.2 & 72.4 & 89.7 & 56 & 50.0 & 67.9 & 76.8 & 50 & 48.0 & 60.0 & 62.0 & 52 & 61.5 & 67.3 & 78.8 \\
\hline All Sildenafil Doses Combined & 77 & 24.7 & 37.7 & 76.6 & 165 & 67.3 & 77.6 & 87.3 & 152 & 64.5 & 72.4 & 75.0 & 154 & 47.4 & 57.1 & 63.6 \\
\hline Low Dose & 24 & 37.5 & 50.0 & 79.2 & 39 & 48.7 & 69.2 & 82.1 & 36 & 52.8 & 66.7 & 66.7 & 37 & 48.6 & 56.8 & 67.6 \\
\hline Medium Dose & 26 & 19.2 & 34.6 & 73.1 & 55 & 74.5 & 80.0 & 85.5 & 49 & 65.3 & 75.5 & 77.6 & 49 & 51.0 & 59.2 & 63.3 \\
\hline High Dose & 27 & 18.5 & 29.6 & 77.8 & 71 & 71.8 & 80.3 & 91.5 & 67 & 70.1 & 73.1 & 77.6 & 68 & 44.1 & 55.9 & 61.8 \\
\hline
\end{tabular}

$\mathrm{VO}_{2}=$ oxygen consumption .

Numbers represent the percentage of patients having less than or equal to the specified percentage change from baseline for each parameter. Table 4: Cumulative Distribution Function Values (\%) at Specified Percent Changes from Baseline. 
Citation: Cappelleri JC, Hwang LJ, Mardekian J, Teal SA, Mychaskiw MA (2013) Cumulative Distribution Functions of Sildenafil Citrate on Exercise Capacity and Hemodynamic Function in Children with Pulmonary Arterial Hypertension. J Pulmon Resp Med S4: 002. doi:10.4172/2161105X.S4-002
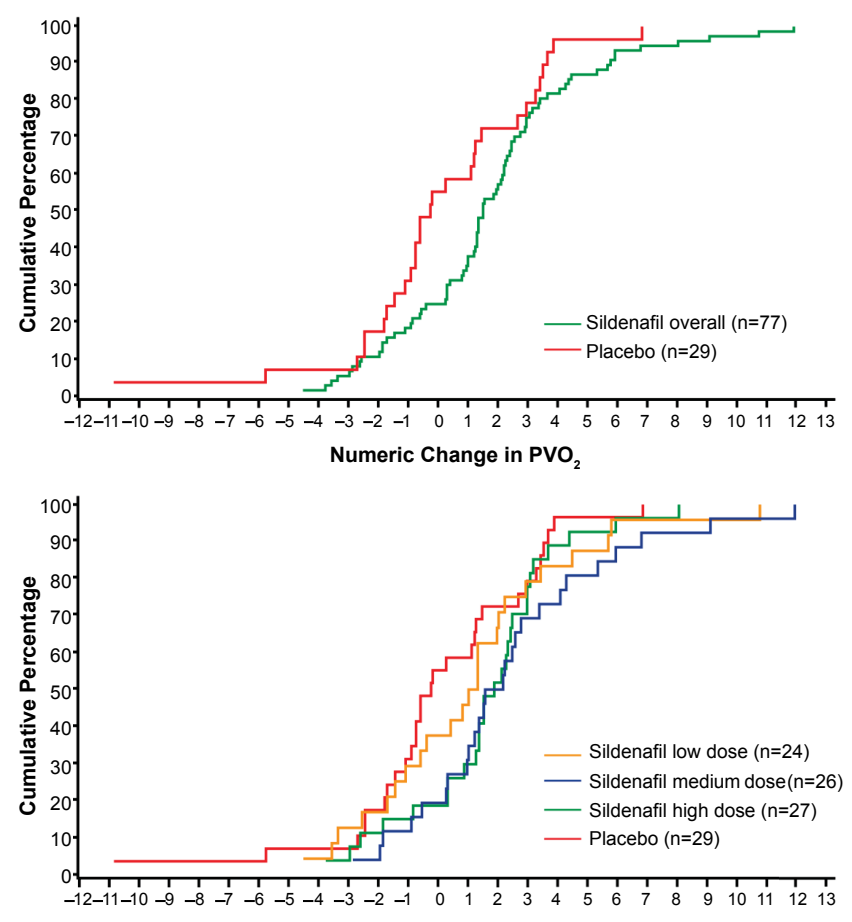

Numeric Change in $\mathrm{PVO}_{2}$

Figure 5: Cumulative Distribution Functions of Numeric Change from Baseline to Week 16 in Peak Volume $\mathrm{O}_{2}$ consumption (PVO2): Active Doses Combined vs. Placebo (Top) and Active Doses Separated vs. Placebo (Bottom).
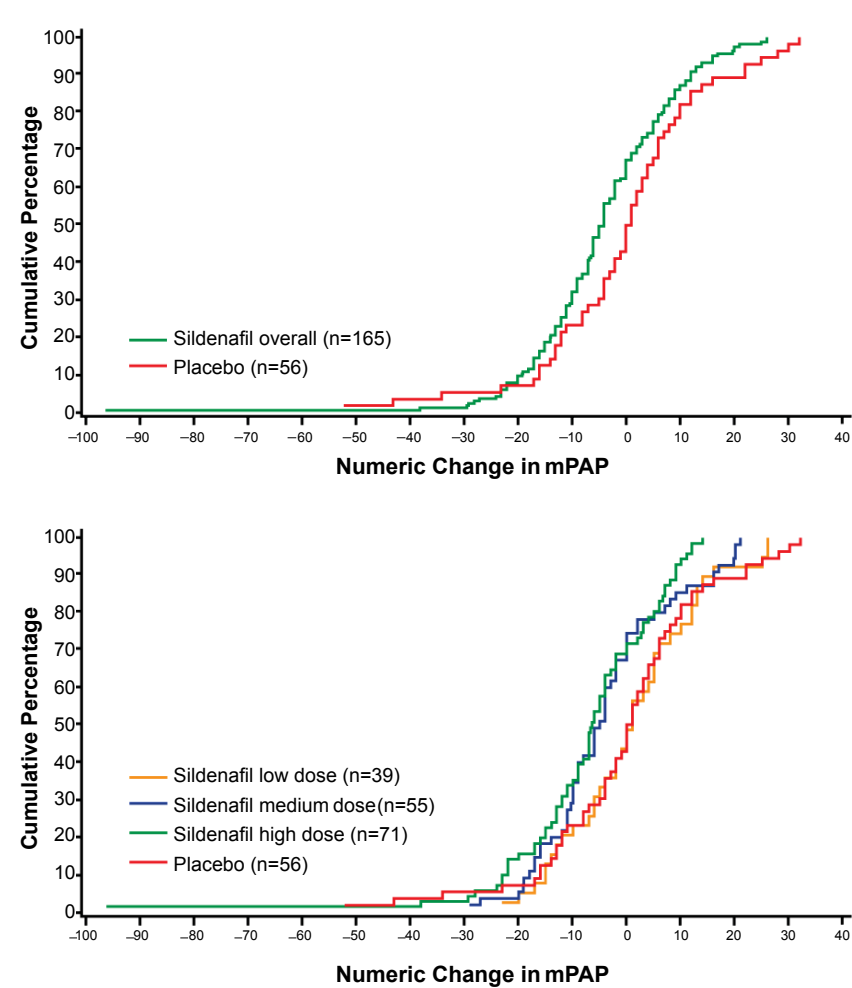

Figure 6: Cumulative Distribution Functions of Numeric Change from Baseline to Week 16 in Mean Pulmonary Arterial Pressure mPAP: Active Doses Combined vs. Placebo (Top) and Active Doses Separated vs. Placebo (Bottom).
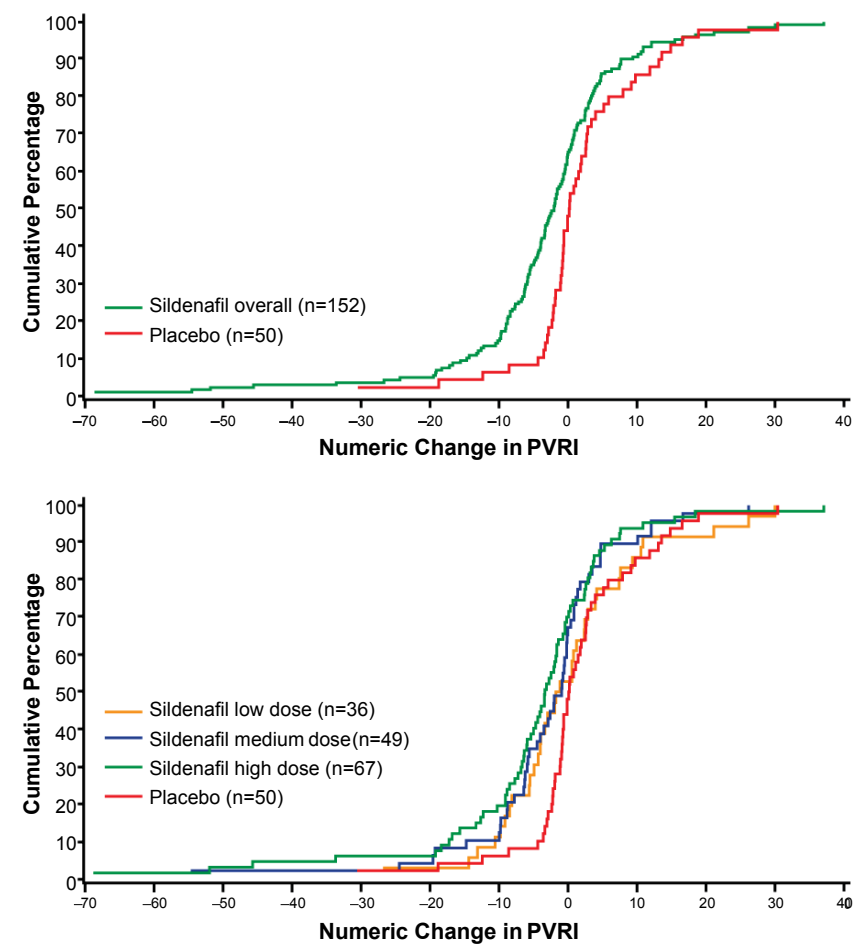

Figure 7: Cumulative Distribution Functions of Numeric Change from Baseline to Week 16 in Pulmonary Vascular Resistance Index (PVRI): Active Doses Combined vs. Placebo (Top) and Active Doses Separated vs. Placebo (Bottom).
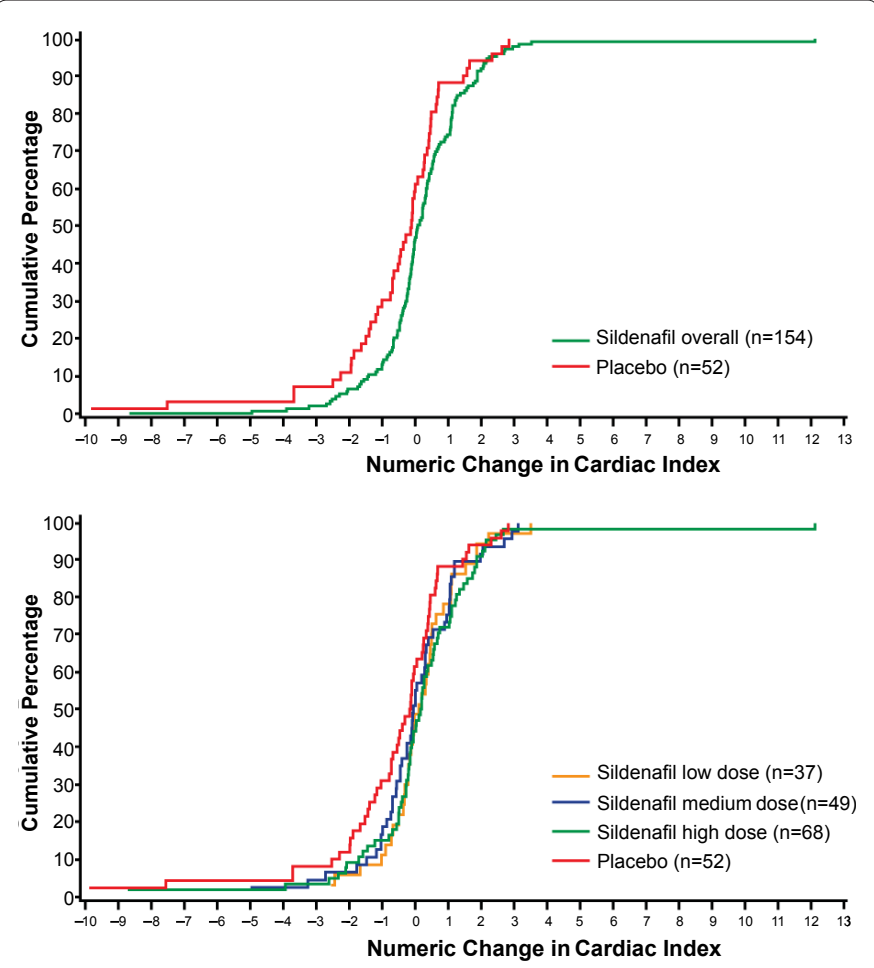

Figure 8: Cumulative Distribution Functions of Numeric Change from Baseline to Week 16 in Cardiac Index: Active Doses Combined vs. Placebo (Top) and Active Doses Separated vs. Placebo (Bottom). 
Citation: Cappelleri JC, Hwang LJ, Mardekian J, Teal SA, Mychaskiw MA (2013) Cumulative Distribution Functions of Sildenafil Citrate on Exercise Capacity and Hemodynamic Function in Children with Pulmonary Arterial Hypertension. J Pulmon Resp Med S4: 002. doi:10.4172/2161105X.S4-002

Page 7 of 8

\begin{tabular}{|c|c|c|c|c|}
\hline \multirow{2}{*}{ Outcome Measure } & \multicolumn{4}{|c|}{ Numeric Change: $\boldsymbol{P}$ Value* vs Placebo } \\
\hline & Overall & Low & Medium & High \\
\hline Peak VO$_{2}$ & 0.02 & 0.60 & 0.06 & 0.02 \\
\hline Mean PAP & 0.05 & 1.0 & 0.04 & 0.01 \\
\hline PVRI & 0.001 & 0.05 & 0.03 & 0.0004 \\
\hline Cardiac index & 0.12 & 0.15 & 0.46 & 0.13 \\
\hline
\end{tabular}

$\mathrm{PAP}=$ pulmonary arterial pressure; $\mathrm{PVRI}=$ pulmonary vascular resistance index; $\mathrm{VO}_{2}=$ oxygen consumption .

*Values are rounded, with those $\leq 0.05$ in bold face.

Table 5: Descriptive $P$ Values (Active Treatment vs. Placebo) for Cumulative Distribution Functions: Numeric Change from Baseline.

\begin{tabular}{|c|c|c|c|c|c|c|c|c|c|c|c|c|c|c|c|c|}
\hline \multirow[b]{2}{*}{ Change from Baseline } & \multicolumn{4}{|c|}{ Peak $\mathrm{VO}_{2}, \mathrm{~mL} / \mathrm{kg} / \mathrm{min}$} & \multicolumn{4}{|c|}{$\begin{array}{l}\text { Mean pulmonary arterial } \\
\text { pressure, } \mathrm{mmHg}\end{array}$} & \multicolumn{4}{|c|}{$\begin{array}{c}\text { Pulmonary vascular resistance } \\
\text { index, Wood units }{ }^{2} \mathbf{m}^{2}\end{array}$} & \multicolumn{4}{|c|}{ Cardiac index, $\mathrm{L} / \mathrm{min} / \mathrm{m}^{2}$} \\
\hline & $\mathbf{n}$ & 0 & 1 & 2 & $\mathbf{n}$ & 0 & 10 & 20 & $\mathbf{n}$ & 0 & 10 & 20 & n & 0 & 1 & 2 \\
\hline Placebo & 29 & 55.2 & 58.6 & 72.4 & 56 & 50.0 & 82.1 & 89.3 & 50 & 48.0 & 86.0 & 98.0 & 52 & 61.5 & 88.5 & 94.2 \\
\hline All Sildenafil Doses Combined & 77 & 24.7 & 37.7 & 57.1 & 165 & 67.3 & 87.3 & 97.6 & 152 & 64.5 & 90.8 & 96.7 & 154 & 47.4 & 74.7 & 92.2 \\
\hline Low Dose & 24 & 37.5 & 50.0 & 70.8 & 39 & 48.7 & 76.9 & 92.3 & 36 & 52.8 & 86.1 & 91.7 & 37 & 48.6 & 78.4 & 94.6 \\
\hline Medium Dose & 26 & 19.2 & 34.6 & 50.0 & 55 & 74.5 & 85.5 & 98.2 & 49 & 65.3 & 89.8 & 98.0 & 49 & 51.0 & 75.5 & 91.8 \\
\hline High Dose & 27 & 18.5 & 29.6 & 51.9 & 71 & 71.8 & 94.4 & 100.0 & 67 & 70.1 & 94.0 & 98.5 & 68 & 44.1 & 72.1 & 91.2 \\
\hline
\end{tabular}

$\mathrm{VO}_{2}=$ oxygen consumption

Numbers represent the percentage of patients having less than or equal to the specified percentage change from baseline for each parameter

Table 6: Cumulative Distribution Function Values (\%) at Specified Numeric Changes from Baseline.

endpoints, such as WHO functional class status and a Physician Global Assessment [29].

Cumulative distribution functions, which incorporate the entire distribution of responses, can lend clinical relevance and enhance interpretation of treatment differences on outcomes for regulatory and scientific purposes. In this article, we report and describe CDFs on exercise capacity and hemodynamic parameters in children with PAH treated with sildenafil in the STARTS-1 study. The distribution of responses for $\mathrm{PVO}_{2}, \mathrm{mPAP}$, PVRI, and cardiac index for sildenafil was descriptively more favorable compared with placebo, suggesting that sildenafil improves exercise capacity and hemodynamic function in children with $\mathrm{PAH}$.

Hemodynamic measures (such as cardiac output and right atrial pressure) as well as exercise capacity (assessed by the 6MWD), are known to be strong predictors of outcome in adult patients with PAH [30,31], while higher PVR is significantly associated with worse survival in children [10]. Whether the short-term improvements in hemodynamics in sildenafil-treated children in STARTS-1 are indicators of longer-term outcome is unknown, and would need to be studied in a controlled setting. More recently in PAH research, larger studies of longer duration have sought to assess the impact of therapies on composite end points including death and significant clinical events. However, to date these studies have been limited to adults. Future research in pediatric PAH should consider the effect of therapies on these longer-term clinical outcome measures.

Cumulative distributions functions can provide insightful, wellrounded, and comprehensive assessments on treatment differences not only for so-called objective or physiological outcomes, such as exercise capacity and hemodynamic parameters, but also for subjective or patient-reported outcomes. A customary challenge when using patientreported outcomes lies in the interpretation of their scores, a challenge that also prevails in the field of pulmonary hypertension, and CDFs can be used to effectively enrich the interpretation of scores on patientreported outcomes as well $[18,32]$. In fact, the FDA final guidance document on patient-reported outcomes supports the use of CDFs to enhance clinical interpretation [23].

In conclusion, $\mathrm{CDFs}$, which incorporate the entire distribution of responses, can enhance clinical interpretation of outcome measures on exercise capacity and hemodynamic function in children with PAH. Within each treatment group, the (cumulative) percentage of patients less than or equal to a percentage (or numeric) change from baseline can be depicted visually and noted descriptively. By CDF analysis, an enhanced profile of sildenafil (vs. placebo) was observed indicating improvement in $\mathrm{PVO}_{2}, \mathrm{mPAP}$ and PVRI, but not cardiac index, in children with PAH.

\section{Acknowledgements}

This research was supported by Pfizer Inc. Simon Teal is an employee of Pfizer Ltd and shareholder of Pfizer Inc. All other authors are employees and shareholders of Pfizer Inc, the manufacturer of sildenafil. Editorial support was provided by Tiffany Brake, PhD, of Complete Healthcare Communications, Inc. and was funded by Pfizer Inc.

\section{References}

1. Galiè N, Torbicki A, Barst R, Dartevelle P, Haworth S, et al. (2004) Guidelines on diagnosis and treatment of pulmonary arterial hypertension. The Task Force on Diagnosis and Treatment of Pulmonary Arterial Hypertension of the European Society of Cardiology. Eur Heart J 25: 2243-2278.

2. Humbert M, Sitbon O, Simonneau G (2004) Treatment of pulmonary arterial hypertension. N Engl J Med 351: 1425-1436.

3. Chen H, Taichman DB, Doyle RL (2008) Health-related quality of life and patient-reported outcomes in pulmonary arterial hypertension. Proc Am Thorac Soc 5: 623-630.

4. Shafazand S, Goldstein MK, Doyle RL, Hlatky MA, Gould MK (2004) Healthrelated quality of life in patients with pulmonary arterial hypertension. Chest 126: $1452-1459$

5. D’Alonzo GE, Barst RJ, Ayres SM, Bergofsky EH, Brundage BH, et al. (1991) Survival in patients with primary pulmonary hypertension. Results from a national prospective registry. Ann Intern Med 115: 343-349.

6. Humbert M, Sitbon O, Chaouat A, Bertocchi M, Habib G, et al. (2006) Pulmonary arterial hypertension in France: results from a national registry. Am J Respir Crit Care Med 173: 1023-1030.

7. Peacock AJ, Murphy NF, McMurray JJ, Caballero L, Stewart S (2007) An epidemiological study of pulmonary arterial hypertension. Eur Respir J 30: 104109.

8. Fraisse $A$, Jais $X$, Schleich JM, di Filippo $S$, Maragnès $P$, et al. (2010) Characteristics and prospective 2-year follow-up of children with pulmonary arterial hypertension in France. Arch Cardiovasc Dis 103: 66-74. 
Citation: Cappelleri JC, Hwang LJ, Mardekian J, Teal SA, Mychaskiw MA (2013) Cumulative Distribution Functions of Sildenafil Citrate on Exercise Capacity and Hemodynamic Function in Children with Pulmonary Arterial Hypertension. J Pulmon Resp Med S4: 002. doi:10.4172/2161 105X.S4-002

Page 8 of 8

9. Berger RM, Beghetti M, Humpl T, Raskob GE, Ivy DD, et al. (2012) Clinical features of paediatric pulmonary hypertension: a registry study. Lancet 379 : 537-546

10. Barst RJ, Ertel SI, Beghetti M, Ivy DD (2011) Pulmonary arterial hypertension: a comparison between children and adults. Eur Respir J 37: 665-677.

11. Galiè N, Hoeper MM, Humbert M, Torbicki A, Vachiery JL, et al. (2009) Guidelines for the diagnosis and treatment of pulmonary hypertension: the Task Force for the Diagnosis and Treatment of Pulmonary Hypertension of the European Society of Cardiology (ESC) and the European Respiratory Society (ERS), endorsed by the International Society of Heart and Lung Transplantation (ISHLT). Eur Heart J 30: 2493-2537.

12. McLaughlin VV, Archer SL, Badesch DB, Barst RJ, Farber HW, et al. (2009) ACCF/AHA 2009 expert consensus document on pulmonary hypertension a report of the American College of Cardiology Foundation Task Force on Expert Consensus Documents and the American Heart Association developed in collaboration with the American College of Chest Physicians; American Thoracic Society, Inc.; and the Pulmonary Hypertension Association. J Am Coll Cardiol 53: 1573-1619.

13. Galiè N, Ghofrani HA, Torbicki A, Barst RJ, Rubin LJ, et al. (2005) Sildenafi citrate therapy for pulmonary arterial hypertension. N Engl J Med 353: 2148 2157

14. Simonneau G, Rubin LJ, Galiè N, Barst RJ, Fleming TR, et al. (2008) Addition of sildenafil to long-term intravenous epoprostenol therapy in patients with pulmonary arterial hypertension: a randomized trial. Ann Intern Med 149: 521 530.

15. Revatio (sildenafil). Summary of Product Characteristics, Pfizer Ltd, Sandwich, UK, 2012.

16. Revatio (sildenafil). Full Prescribing Information, Pfizer, New York, NY, 2012.

17. Barst RJ, Ivy DD, Gaitan G, Szatmari A, Rudzinski A, et al. (2012) A randomized double-blind, placebo-controlled, dose-ranging study of oral sildenafil citrate in treatment-naive children with pulmonary arterial hypertension. Circulation 125 324-334.

18. McLeod LD, Coon CD, Martin SA, Fehnel SE, Hays RD (2011) Interpreting patient-reported outcome results: US FDA guidance and emerging methods. Expert Rev Pharmacoecon Outcomes Res 11: 163-169.

19. Snapinn SM, Jiang Q (2007) Responder analyses and the assessment of a clinically relevant treatment effect. Trials 8: 31 .

20. Senn S (2003) Disappointing dichotomies. Pharmaceut Statist. 2: 239-240.
21. Uryniak T, Chan ISF, Fedorov VV, Jiang Q, Oppenheimer L, et al (2011) Responder analyses - a PhRMA position paper. Stat Biopharm Res. 3: 476 487

22. Conover WJ (1999) Practical Nonparametric Statistics. (3 Edn), John Wiley \& Sons, Hoboken, NJ.

23. US Food and Drug Administration (2009) Guidance for industry. Patientreported outcome measures: use in medical product development to support labeling claims. Fed Regist. 74: 65132-65133.

24. Cai QC, Xu QF, Xu JM, Guo Q, Cheng X, et al. (2006) Refined estimate of the incubation period of severe acute respiratory syndrome and related influencing factors. Am J Epidemiol 163: 211-216

25. Tarassenko L, Clifton DA, Pinsky MR, Hravnak MT, Woods JR, et al. (2011) Centile-based early warning scores derived from statistical distributions of vital signs. Resuscitation 82: 1013-1018.

26. Lammers AE, Hislop AA, Flynn Y, Haworth SG (2008) The 6-minute walk test normal values for children of 4-11 years of age. Arch Dis Child 93: 464-468.

27. Smith G, Reyes JT, Russell JL, Humpl T (2009) Safety of maximal cardiopulmonary exercise testing in pediatric patients with pulmonary hypertension. Chest 135: 1209-1214.

28. Miyamoto S, Nagaya N, Satoh T, Kyotani S, Sakamaki F, et al. (2000) Clinica correlates and prognostic significance of six-minute walk test in patients with primary pulmonary hypertension. Comparison with cardiopulmonary exercise testing. Am J Respir Crit Care Med 161: 487-492.

29. Cappelleri JC, Hwang LJ, Mardekian J, Mychaskiw MA (2012) Assessmen of measurement properties of peak $\mathrm{VO}(2)$ in children with pulmonary arterial hypertension. BMC Pulm Med 12: 54

30. Benza RL, Miller DP, Gomberg-Maitland M, Frantz RP, Foreman AJ, et al (2010) Predicting survival in pulmonary arterial hypertension: insights from the Registry to Evaluate Early and Long-Term Pulmonary Arterial Hypertension Disease Management (REVEAL). Circulation 122: 164-172.

31. Humbert M, Sitbon O, Chaouat A, Bertocchi M, Habib G, et al. (2010) Survival in patients with idiopathic, familial, and anorexigen-associated pulmonary arterial hypertension in the modern management era. Circulation 122: 156-163.

32. Cappelleri JC, Zou KH, Bushmakin AG, Carlsson MO, Symonds T (2013) Cumulative response curves to enhance interpretation of treatment differences on the Self-Esteem And Relationship questionnaire for men with erectile dysfunction. BJU Int 111: E115-120.
This article was originally published in a special issue, Pulmonary Hypertension handled by Editor(s). Dr. Zeenat Safdar, Baylor College of Medicine, United States 